\title{
Comparative Effectiveness of Revascularization Strategies
}

\author{
William S. Weintraub, M.D., Maria V. Grau-Sepulveda, M.D., M.P.H., Jocelyn M. Weiss, \\ Ph.D., M.P.H., Sean M. O'Brien, Ph.D., Eric D. Peterson, M.D., M.P.H., Paul Kolm, Ph.D., \\ Zugui Zhang, Ph.D., Lloyd W. Klein, M.D., Richard E. Shaw, Ph.D., Charles McKay, M.D., \\ Laura L. Ritzenthaler, M.B.A., Jeffrey J. Popma, M.D., John C. Messenger, M.D., David M. \\ Shahian, M.D., Frederick L. Grover, M.D., John E. Mayer, M.D., Cynthia M. Shewan, Ph.D., \\ Kirk N. Garratt, M.D., Issam D. Moussa, M.D., George D. Dangas, M.D., and Fred H. \\ Edwards, M.D. \\ Christiana Care Health System, Newark, DE (W.S.W., P.K., Z.Z.); Duke Clinical Research \\ Institute, Durham, NC (M.V.G.-S., S.M.O., E.D.P.); the American College of Cardiology, \\ Washington, DC (J.M.W., L.L.R.); Advocate Illinois Masonic Medical Center (L.W.K.) and the \\ Society of Thoracic Surgeons (C.M.S.) - both in Chicago; California Pacific Medical Center, San \\ Francisco (R.E.S.); Harbor-UCLA Medical Center, Torrance, CA (C.M.); Beth Israel Deaconess \\ Medical Center (J.J.P.), Massachusetts General Hospital (D.M.S.), and Children's Hospital \\ Boston (J.E.M.) - all in Boston; the University of Colorado School of Medicine, Aurora (J.C.M., \\ F.L.G.); Denver Veterans Affairs Medical Center, Denver (F.L.G.); Lenox Hill Heart and Vascular \\ Institute of New York (K.N.G.) and Mount Sinai Medical Center (G.D.D.) - both in New York; and \\ the Mayo Clinic (I.D.M.) and the University of Florida (F.H.E.) - both in Jacksonville
}

\section{Abstract}

BACKGROUND-Questions persist concerning the comparative effectiveness of percutaneous coronary intervention (PCI) and coronary-artery bypass grafting (CABG). The American College of Cardiology Foundation (ACCF) and the Society of Thoracic Surgeons (STS) collaborated to compare the rates of long-term survival after PCI and CABG.

METHODS-We linked the ACCF National Cardiovascular Data Registry and the STS Adult Cardiac Surgery Database to claims data from the Centers for Medicare and Medicaid Services for the years 2004 through 2008. Outcomes were compared with the use of propensity scores and inverse-probability-weighting adjustment to reduce treatment-selection bias.

RESULTS-Among patients 65 years of age or older who had two-vessel or three-vessel coronary artery disease without acute myocardial infarction, 86,244 underwent CABG and 103,549 underwent PCI. The median follow-up period was 2.67 years. At 1 year, there was no significant difference in adjusted mortality between the groups (6.24\% in the CABG group as compared with $6.55 \%$ in the PCI group; risk ratio, 0.95 ; $95 \%$ confidence interval [CI], 0.90 to 1.00). At 4 years, there was lower mortality with CABG than with PCI ( $16.4 \%$ vs. $20.8 \%$; risk ratio, $0.79 ; 95 \% \mathrm{CI}, 0.76$ to 0.82 ). Similar results were noted in multiple subgroups and with the

Address reprint requests to Dr. Weintraub at the Section of Cardiology, Christiana Care Health System, 4755 Ogletown-Stanton Rd., Newark, DE 19718, or at wweintraub@christianacare.org.

No other potential conflict of interest relevant to this article was reported.

Disclosure forms provided by the authors are available with the full text of this article at NEJM.org. 
use of several different analytic methods. Residual confounding was assessed by means of a sensitivity analysis.

CONCLUSIONS-In this observational study, we found that, among older patients with multivessel coronary disease that did not require emergency treatment, there was a long-term survival advantage among patients who underwent CABG as compared with patients who underwent PCI. (Funded by the National Heart, Lung, and Blood Institute.)

The strategies of percutaneous coronary intervention (PCI) and coronary-artery bypass grafting (CABG) for revascularization have been compared in randomized clinical trials. ${ }^{1,2}$ Although the best way to control for treatment-selection bias is to conduct a randomized trial, such trials often have limited power to evaluate subgroups, and the results may not be generalizable, since patients and centers are often highly selected. Nonrandomized, observational data from clinical databases can complement data from clinical trials, because observational data, if they are from a larger and more representative population, may better reflect real-world practice.

The American College of Cardiology Foundation (ACCF) and the Society of Thoracic Surgeons (STS) developed a partnership, the ACCF and STS Database Collaboration on the Comparative Effectiveness of Revascularization Strategies (ASCERT), to compare the outcomes of PCI and CABG, using information from records in their respective databases, with follow-up data from claims records of the Centers for Medicare and Medicaid Services (CMS).

\section{METHODS}

\section{STUDY OVERSIGHT}

The authors designed the ASCERT study. The data were collected at the participating institutions of the STS and ACCF databases and were assembled and analyzed by the authors, who vouch for the accuracy of the data and all analyses. An independent institutional review board approved the study and waived the requirement for informed consent.

\section{STUDY POPULATION}

The process of selecting the study population began with the identification of CMS claims for either CABG or PCI with hospital discharge dates between January 1, 2004, and December 31, 2007, from sites participating in both the ACCF PCI database (CathPCI Registry) and the STS Adult Cardiac Surgery Database (ACD). The CathPCI Registry and the ACD were linked to CMS claims files by probabilistic matching, thus circumventing the need for universal patient identifiers. ${ }^{3}$ Records from the clinical and CMS databases were considered to represent the same patient if they were fully matched with respect to a set of indirect identifiers, including the patient's date of birth, sex, hospital identification number, admission date, and discharge date.

Patients were excluded from the study if they met any of the following criteria: single-vessel disease, left main coronary artery disease, cardiogenic shock within 24 hours before CABG 
or at the time of admission to the hospital for PCI, myocardial infarction within 7 days before $\mathrm{CABG}$ or before admission to the hospital for PCI, insertion of an intraaortic balloon pump before either procedure, or CABG or valve surgery or PCI within 180 days before the current admission. If a report of $\mathrm{CABG}$ and a report of PCI were both associated with the same CMS claim record, the patient was considered to have undergone PCI followed by CABG. Only the first eligible revascularization record for each patient was analyzed.

\section{ADJUSTMENT FOR DIFFERENCES BETWEEN GROUPS}

It was anticipated that the PCI study population and the CABG study population would differ substantially with respect to preprocedural characteristics. We therefore collected information on baseline variables that were available in both registries to make adjusted comparisons feasible. Variables common to both registries were identified from versions 2.41 and 2.52 of the data specifications for the ACD and versions 2 and 3 of the data specifications for the CathPCI Registry. We imputed missing values of continuous variables by stratifying patients according to treatment group and combinations of related risk factors and then imputing stratum-specific medians. Missing categorical variables were imputed to the most common category. Additional details regarding the approach to imputation are provided in the Supplementary Appendix, available with the full text of this article at NEJM.org.

Propensity scores to estimate the probability, on the basis of patient and hospital characteristics, that patients would be selected for CABG were developed with the use of logistic regression to adjust for between-group differences in baseline characteristics of the patients and hospitals. ${ }^{4}$ Details of the individual variables included in the propensity model are provided in the Supplementary Appendix. Inverse probability weighting that was based on the propensity score was then used as the primary tool to adjust for differences between the two treatment groups. ${ }^{5}$ This approach, which was implemented to create balance, involved weighting each patient who underwent $\mathrm{CABG}$ by the inverse of the probability that he or she would be selected for CABG and weighting each patient who underwent PCI by the inverse of the probability that he or she would be selected for PCI. We verified the performance of the propensity model by comparing the distribution of covariates and propensity scores between treatment groups both before and after inverse probability weighting. ${ }^{6}$

\section{STATISTICAL ANALYSIS}

Summary statistics are presented as percentages in the case of categorical variables and as means with standard deviations in the case of continuous variables. Baseline characteristics of the patients were compared between treatment groups with the use of the Pearson chisquare test for categorical variables and the Wilcoxon rank-sum test for continuous variables.

The primary end point was all-cause mortality, which was identified from information in the CMS database. Patients were followed from the date of the index revascularization through December 31, 2008. Unadjusted survival curves were estimated with the use of the KaplanMeier $\operatorname{method}^{7}$; adjusted survival curves were estimated with the use of the inverse- 
probability-weighting approach of Cole and Hernan. ${ }^{8}$ For each treatment group, the survival curves adjusted with the use of inverse probability weighting represent the expected rate of survival if the treatment of interest (PCI or CABG) were applied to all study patients. Using estimated rates of survival among patients undergoing PCI and among those undergoing $\mathrm{CABG}$, we calculated risk ratios at specific time points and used bootstrap methods to obtain 95\% confidence intervals. The comparison of CABG with PCI was performed in the overall population and in prospectively defined subgroups.

Several sensitivity analyses were performed (as described in the Supplementary Appendix). Survival curves were re-estimated separately for CABG and PCI with the use of Cox proportional-hazard models without propensity scores. ${ }^{9}$ Covariates for each model were identical to those in the propensity model described above. Using these models, we estimated the average survival curves that would be predicted if all the patients in the study were to undergo PCI and if all the patients were to undergo CABG. We also combined inverse probability weighting and model-based approaches for a "doubly robust" analysis. ${ }^{10}$ In addition, we conducted a sensitivity analysis using data from patients matched with respect to the propensity score.

We also explored the effect of potential unmeasured confounders. We developed covariateadjusted Cox models to estimate hazard ratios for CABG as compared with PCI. Even if the proportional-hazards assumption is not met for the treatment-group variable, the hazard ratio may be interpreted as an "average" over the observed event times. ${ }^{9}$ We then used the method of Lin et al. ${ }^{11}$ to assess whether the observed differences in the rate of death could be fully explained by an unmeasured confounder.

\section{RESULTS}

\section{CHARACTERISTICS OF THE STUDY POPULATION}

A total of 1,542,872 claims for PCI and 581,036 claims for CABG, for 1,943,653 unique patients, were recorded in the CMS database between January 1, 2004, and December 31, 2007, at 644 sites participating in both the CathPCI Registry and the ACD. After the exclusion criteria were applied, data from 103,549 patients who underwent PCI ( $7 \%$ of the total) and 86,244 patients who underwent CABG (15\% of the total) were included in the analysis (Fig. 1 in the Supplementary Appendix).

Table 1 shows selected baseline characteristics of the study patients (a list of all variables is provided in Table 1 in the Supplementary Appendix). Before adjustment with the use of inverse probability weighting, the patients undergoing PCI, as compared with those undergoing $\mathrm{CABG}$, were, on average, older, and more patients were women. More patients in the CABG group than in the PCI group had heart failure, diabetes, hypertension, chronic lung disease, cerebrovascular disease, a history of smoking, or peripheral arterial disease. More patients in the PCI group than in the CABG group had prior myocardial infarction or unstable angina or required urgent procedures. The ejection fraction was somewhat higher in the PCI group. The largest difference between the groups was in the distribution of the number of diseased vessels, with patients in the PCI group more often having two-vessel disease and patients in the CABG group more often having three-vessel disease. After 
adjustment with the use of inverse probability weighting, all the clinical covariates were well balanced (Table 1). Among patients who underwent PCI, 78\% received drug-eluting stents, $16 \%$ received bare-metal stents, and $6 \%$ underwent the procedure without the placement of stents.

As expected, patients in the PCI group had a lower probability of being selected for CABG than did those in the $\mathrm{CABG}$ group (Fig. 1), with the median and interquartile range of the propensity scores for CABG reflecting this difference (PCI group: median, 20.3\%; interquartile range, 9.9 to 44.7 ; CABG group: median, $71.3 \%$; interquartile range, 50.1 to 85.1).

\section{OUTCOMES}

The follow-up time ranged from 1 to 5 years (average follow-up: overall, 2.72 years; CABG group, 2.82 years, and PCI group, 2.63 years; median follow-up: overall, 2.67 years; CABG group, 2.83 years, and PCI group, 2.53 years). Unadjusted survival curves are shown in Figure 2, and the survival curves adjusted with the use of inverse probability weighting are shown in Figure 3. At 1 year, there was no significant difference in adjusted mortality between the groups (6.2\% in the CABG group as compared with $6.6 \%$ in the PCI group; risk ratio, $0.95 ; 95 \%$ confidence interval $[\mathrm{CI}], 0.90$ to 1.00 ). The adjusted 4 -year mortality was $16.4 \%$ in the CABG group and $20.8 \%$ in the PCI group (risk ratio, $0.79 ; 95 \% \mathrm{CI}, 0.76$ to 0.82 ). Sensitivity analyses performed with the use of the Cox model and with the use of data from propensity-matched groups yielded similar results (Table 1 and Fig. 2 in the Supplementary Appendix). The 4-year risk ratios showed a benefit of CABG across subgroups defined according to sex, age, presence or absence of diabetes, body-mass index, presence or absence of chronic lung disease, ejection fraction, and glomerular filtration rate (Fig. 3 in the Supplementary Appendix) and in both a high-risk group and a low-risk group. CABG was also associated with a benefit across subgroups defined according to quintile of propensity score for $\mathrm{CABG}$ (from the lowest quintile, 0 to $20 \%$, to the highest quintile, 80 to $100 \%$ ). Thus, the rate of survival was better with CABG even among patients whose propensity scores were most consistent with selection for PCI.

\section{EFFECT OF UNMEASURED CONFOUNDING}

The estimated average hazard ratio with CABG as compared with PCI in the Cox-model analysis was similar to the estimated 4-year risk ratio derived with the use of inverse probability weighting (covariate-adjusted hazard ratio, $0.79 ; 95 \%$ CI, 0.76 to 0.82 ). Figure 4 shows the method that can be used to determine whether an unmeasured binary risk factor could explain a hazard ratio of this magnitude. The $\mathrm{x}$ axis represents the hypothetical prevalence of the unmeasured confounder in the PCI population, and the y axis represents the hypothetical hazard ratio for mortality associated with this confounder. The curved lines indicate the hypothetical prevalence $(5 \%, 10 \%, 20 \%, 30 \%$, or $40 \%)$ of the potential confounder in the $\mathrm{CABG}$ group. For example, if an unmeasured risk factor was present in $10 \%$ of the patients in the CABG group (green curved line) and in $20 \%, 35 \%$, or $50 \%$ of the patients in the PCI group, then the hazard ratio that would be required for an unmeasured confounder to account for the observed decreased risk with CABG (i.e., to shift the upper $95 \%$ confidence interval from 0.80 to 1.00 ) would be $4.25,2.09$, and 1.65 , respectively. 
Similarly, if an unmeasured risk factor was present in $20 \%$ of the patients in the CABG group (dark blue curved line) and in $30 \%, 45 \%$, or $60 \%$ of the patients in the PCI group, then the hazard ratio that would be required for an unmeasured risk factor to account for the observed increased risk with PCI would be 5.82, 2.22, and 1.70, respectively.

A single unmeasured confounder could produce the observed survival differences only if it increased the long-term risk of death by a factor of approximately two or if the long-term risk of death was three to five times as high in the PCI group as in the CABG group. As an example of a potential unmeasured confounder, suppose that patient frailty (yes or no) could be assessed in our study. If frailty was present in $10 \%$ of the patients in the CABG group (green curved line) but in 35\% of patients in the PCI group (x axis), and if frailty increased the risk of death by a factor of slightly more than two (hazard ratio, 2.09), then frailty alone could itself account for the observed difference in mortality between the study groups.

\section{DISCUSSION}

The ASCERT study was a collaborative outcome study in which data from the STS and ACCF registries were used to evaluate the effectiveness of revascularization with CABG as compared with PCI. In this study, we found that among Medicare patients 65 years of age or older with ischemic heart disease that required revascularization on a nonemergency basis, there was no significant difference in adjusted mortality at 1 year between patients who had undergone CABG and those who had undergone PCI, but mortality at 4 years was lower in the CABG group than in the PCI group. These findings were noted in all subgroups. The survival rate was better with $\mathrm{CABG}$ even among patients whose propensity scores were most consistent with selection for PCI.

Our findings should be evaluated in the context of results from other studies. There have been seven randomized, controlled trials comparing CABG with balloon angioplasty, ${ }^{12-18}$ four comparing CABG with PCI and placement of bare-metal stents, ${ }^{19-22}$ and one comparing $\mathrm{CABG}$ with $\mathrm{PCI}$ and placement of drug-eluting stents. ${ }^{2} \mathrm{~A}$ survival advantage with CABG was seen in the Stent or Surgery trial (SoS; ClinicalTrials.gov number, NCT00475449) ${ }^{23}$ and in the subgroup with treated diabetes in the Bypass Angioplasty Revascularization Intervention trial (BARI, NCT00000462) ${ }^{24}$ A meta-analysis of these trials, which included 7812 patients, showed a trend toward a survival advantage with CABG. ${ }^{1}$ In a subgroup analysis according to the presence or absence of diabetes, there was a survival advantage with CABG among patients who had diabetes, whereas there was no significant advantage among patients who did not have diabetes. Among patients younger than 55 years of age, there was a trend toward a benefit with PCI as compared with CABG, whereas among patients older than 65 years of age, mortality was significantly higher with PCI. This meta-analysis did not include the Synergy between PCI with Taxus and Cardiac Surgery trial (SYNTAX, NCT00114972), a randomized trial in which contemporary methods of revascularization were used. At 3 years, among patients in the SYNTAX trial who had three-vessel disease, both the overall rate of death and the rate of death from cardiac causes were significantly lower among patients who had undergone CABG than among those who had undergone PCI. ${ }^{25}$ The mortality was substantially lower in the SYNTAX trial than in the present study, possibly because the patients in the SYNTAX trial 
were younger, may have had fewer coexisting conditions, or may have had less severe disease.

Six previous observational studies, three of which were multicenter studies ${ }^{26-28}$ and three of which were single-center studies, ${ }^{29-31}$ also showed results similar to those presented here. The consistent findings in these observational studies lend support to the finding of a survival advantage with CABG observed in our study. Whereas the multicenter studies focused on a single state ${ }^{26,27}$ or region $^{28}$ in the United States, the national scope of the current study indicates that the favorable survival rates observed among those selected for CABG extend across the entire United States.

The ASCERT study shows the potential benefits of linking large clinical and administrative databases to assess the comparative effectiveness of therapies in large patient populations. Perhaps the most compelling advantage of this approach is the ability to evaluate outcomes in broadly representative patient populations rather than the selected population of a randomized, controlled trial. Continually developing new randomized, controlled trials comparing PCI with CABG with each advancement in technology is not feasible, but data that are linked as they are in this project can be readily collected on an ongoing basis to provide continuity for subsequent studies.

This study also shows the specific advantages of linking clinical and administrative databases. Clinical databases are well suited to risk adjustment and the identification of clinically important subgroups but lack information on long-term outcomes. Administrative data sets have limited capacity for clinical considerations, but they provide information on long-term outcomes. Linking clinical data with administrative data capitalizes on the advantages of each.

There are several limitations of this study. Despite efforts by the STS and ACCF to provide coordinated, harmonized data records, there are limitations of the data that, in turn, limit the ability to match data across the registries and lead to some uncertainty regarding the interpretation of variables that could affect the results. The elapsed time from the onset of an event was tabulated differently for some of the variables, resulting in some small differences in definitions between the databases. The angiographic data in the STS and ACCF registries are not as detailed as they are in contemporary trials such as SYNTAX; therefore, the ability to establish balance with respect to angiographic variables was limited. No large clinical databases contain the level of anatomical detail required to calculate the SYNTAX score. Data on coronary flow reserve or other functional or anatomical data may supplant strictly angiographic data in the future.

The ASCERT trial is an observational study, and the unadjusted clinical profile and propensity scores for $\mathrm{CABG}$ differed between the treatment groups. Although adjustment with the use of inverse probability weighting resulted in excellent balance between the CABG and PCI populations, the potential remains for unmeasured confounders to have influenced the findings. Some variables that are known in clinical practice to have a profound effect on the choice of revascularization (e.g., extensive coronary disease, the presence of chronic total coronary occlusions, and patient frailty) were not available for this 
analysis. Although the sensitivity analysis suggests that either a single powerful variable or several confounding variables acting in concert could conceivably account for the betweengroup difference in the rate of survival, such confounders could also increase the difference. ${ }^{32,33}$

Our study also has analytic limitations. Data were missing for certain variables — in particular, the glomerular filtration rate and ejection fraction. In addition, probabilistic matching may not be as reliable a method for finding matches across databases as is the use of a universal patient identifier, which was not available. Finally, the study population consisted entirely of Medicare patients; therefore, the results may not be generalizable to younger patients.

In summary, the ASCERT study used data from the ACCF PCI database and the STS $\mathrm{CABG}$ database, with linkage to CMS claims records, to evaluate the comparative effectiveness of PCI and CABG. We found that among patients older than 65 years of age with multivessel coronary artery disease that did not require emergency treatment, there was a long-term survival advantage associated with CABG as compared with PCI.

\section{Supplementary Material}

Refer to Web version on PubMed Central for supplementary material.

\section{Acknowledgments}

The content of this article is solely the responsibility of the authors and does not necessarily represent the official views of the National Heart, Lung, and Blood Institute or the National Institutes of Health, the Society of Thoracic Surgeons, or the American College of Cardiology Foundation.

Supported by a grant (RC2HL101489) from the National Heart, Lung, and Blood Institute.

Dr. Popma reports receiving consulting fees from Abbott Vascular, Boston Scientific, and Covidien and grant support from Abbott Vascular, Abiomed, Boston Scientific, Cordis, and Medtronic; Dr. Messenger, receiving grant support from Medtronic; Dr. Mayer, receiving honoraria and reimbursement for travel expenses from CHMC Cardiovascular Surgical Foundation; Dr. Dangas, receiving consulting fees from Abbott Vascular, Astra-Zeneca, Eli Lilly, Johnson \& Johnson, and Ogilvy, providing expert testimony regarding stroke in a patient after cardioversion for atrial fibrillation and regarding infection after heart-valve implantation, and receiving grant support from Bristol-Myers Squibb, Eli Lilly, Daichi-Sankyo, the Medicines Company, and Sanofi-Aventis, lecture fees from Abbott Vascular, AstraZeneca, Boston Scientific, Bracco, Bristol-Myers Squibb, Guerbet, Eli Lilly, Johnson \& Johnson, the Medicines Company, and Sanofi-Aventis, royalties from Wiley and Informa, and reimbursement for travel expenses from the Cardiovascular Research Foundation; and Dr. Edwards, being an employee of the Society of Thoracic Surgeons.

\section{References}

1. Hlatky MA, Boothroyd DB, Bravata DM, et al. Coronary artery bypass surgery compared with percutaneous coronary interventions for multivessel disease: a collaborative analysis of individual patient data from ten randomised trials. Lancet. 2009; 373:1190-7. [PubMed: 19303634]

2. Serruys PW, Morice MC, Kappetein AP, et al. Percutaneous coronary intervention versus coronaryartery bypass grafting for severe coronary artery disease. N Engl J Med. 2009; 360:961-72. [PubMed: 19228612]

3. Douglas PS, Brennan JM, Anstrom KJ, et al. Clinical effectiveness of coronary stents in elderly persons: results from 262,700 Medicare patients in the American College of Cardiology-National Cardiovascular Data Registry. J Am Coll Cardiol. 2009; 53:1629-41. [PubMed: 19406337] 
4. Rosenbaum PR, Rubin D. The central role of propensity score in observation studies for causal effects. Biometrika. 1983; 70:41-55.

5. Curtis LH, Hammill BG, Eisenstein EL, Kramer JM, Anstrom KJ. Using inverse probabilityweighted estimators in comparative effectiveness analyses with observational databases. Med Care. 2007; 45(Suppl 2):S103-S107. [PubMed: 17909367]

6. Rosenbaum PR. Model-based direct adjustment. J Am Stat Assoc. 1987; 82:387-94.

7. Kaplan EL, Meier P. Nonparametric estimation from incomplete observations. J Am Stat Assoc. 1958; 53:457-81.

8. Cole SR, Hernan MA. Adjusted survival curves with inverse probability weights. Comput Methods Programs Biomed. 2004; 75:45-9. [PubMed: 15158046]

9. Therneau, TM.; Grambsch, PM. Modeling survival data: extending the Cox model. New York: Springer; 2000.

10. Hirano K, Imbens G. Estimation of causal effects using propensity score weighting: an application to data on right heart catheterization. Health Serv Outcomes Res Methodol. 2001; 2:259-78.

11. Lin DY, Psaty BM, Kronmal RA. Assessing the sensitivity of regression results to unmeasured confounders in observational studies. Biometrics. 1998; 54:948-63. [PubMed: 9750244]

12. King SB III, Lembo NJ, Weintraub WS, et al. A randomized trial comparing coronary angioplasty with coronary bypass surgery. N Engl J Med. 1994; 331:1044-50. [PubMed: 8090163]

13. Hamm CW, Reimers J, Ischinger T, Rupprecht HJ, Berger J, Bleifeld W. A randomized study of coronary angioplasty compared with bypass surgery in patients with symptomatic multivessel coronary disease. N Engl J Med. 1994; 331:1037-43. [PubMed: 8090162]

14. First-year results of CABRI (Coronary Angioplasty versus Bypass Revascularisation Investigation). Lancet. 1995; 346:1179-84. [PubMed: 7475656]

15. Coronary angioplasty versus coronary artery bypass surgery: the Randomized Intervention Treatment of Angina (RITA) trial. Lancet. 1993; 341:573-80. [PubMed: 8094826]

16. Rodriguez A, Boullon F, Perez-Baliño N, Paviotti C, Liprandi MI, Palacios IF. Argentine randomized trial of percutaneous transluminal coronary angioplasty versus coronary artery bypass surgery in multivessel disease (ERACI): in-hospital results and 1-year follow-up. J Am Coll Cardiol. 1993; 22:1060-7. [PubMed: 8409041]

17. The Bypass Angioplasty Revascularization Investigation (BARI) Investigators. Comparison of coronary bypass surgery with angioplasty in patients with multivessel disease. N Engl J Med. 1996; 335:217-25. Erratum, N Engl J Med 1997; 336:147. [PubMed: 8657237]

18. Carrié D, Elbaz M, Puel J, et al. Five-year outcome after coronary angioplasty versus bypass surgery in multivessel coronary artery disease: results from the French Monocentric Study. Circulation. 1997; 96(Suppl):II-1-II-6.

19. Serruys PW, Unger F, Sousa JE, et al. Comparison of coronary-artery bypass surgery and stenting for the treatment of multivessel disease. N Engl J Med. 2001; 344:1117-24. [PubMed: 11297702]

20. SoS Investigators. Coronary artery bypass surgery versus percutaneous coronary intervention with stent implantation in patients with multivessel coronary artery disease (the Stent or Surgery trial): a randomised controlled trial. Lancet. 2002; 360:965-70. [PubMed: 12383664]

21. Rodriguez A, Bernardi V, Navia J, et al. Argentine randomized study: coronary angioplasty with stenting versus coronary bypass surgery in patients with multiple-vessel disease (ERACI II): 30day and one-year follow-up results. J Am Coll Cardiol. 2001; 37:51-8. Erratum, J Am Coll Cardiol 2001; 37: 973-4. [PubMed: 11153772]

22. Hueb W, Lopes NH, Gersh BJ, et al. Five-year follow-up of the Medicine, Angioplasty, or Surgery Study (MASS II): a randomized controlled clinical trial of 3 therapeutic strategies for multivessel coronary artery disease. Circulation. 2007; 115:1082-9. [PubMed: 17339566]

23. Booth J, Clayton T, Pepper J, et al. Randomized, controlled trial of coronary artery bypass surgery versus percutaneous coronary intervention in patients with multivessel coronary artery disease: six-year follow-up from the Stent or Surgery Trial (SoS). Circulation. 2008; 118:381-8. [PubMed: 18606919]

24. Seven-year outcome in the Bypass Angioplasty Revascularization Investigation (BARI) by treatment and diabetic status. J Am Coll Cardiol. 2000; 35:1122-9. [PubMed: 10758950] 
25. Kappetein AP, Feldman TE, Mack MJ, et al. Comparison of coronary bypass surgery with drugeluting stenting for the treatment of left main and/or three-vessel disease: 3-year follow-up of the SYNTAX trial. Eur Heart J. 2011; 32:2125-34. [PubMed: 21697170]

26. Hannan EL, Racz MJ, Walford G, et al. Long-term outcomes of coronary-artery bypass grafting versus stent implantation. N Engl J Med. 2005; 352:2174-83. [PubMed: 15917382]

27. Hannan EL, Wu C, Walford G, et al. Drug-eluting stents vs. coronary-artery bypass grafting in multivessel coronary disease. N Engl J Med. 2008; 358:331-41. [PubMed: 18216353]

28. Malenka DJ, Leavitt BJ, Hearne MJ, et al. Comparing long-term survival of patients with multivessel coronary disease after CABG or PCI: analysis of BARI-like patients in northern New England. Circulation. 2005; 112(Suppl):I-371-I-376. [PubMed: 16159849]

29. Brener SJ, Lytle BW, Casserly IP, Schneider JP, Topol EJ, Lauer MS. Propensity analysis of longterm survival after surgical or percutaneous revascularization in patients with multivessel coronary artery disease and high-risk features. Circulation. 2004; 109:2290-5. [PubMed: 15117846]

30. Smith PK, Califf RM, Tuttle RH, et al. Selection of surgical or percutaneous coronary intervention provides differential longevity benefit. Ann Thorac Surg. 2006; 82:1420-9. [PubMed: 16996946]

31. van Domburg RT, Takkenberg JJM, Noordzij LJ, et al. Late outcome after stenting or coronary artery bypass surgery for the treatment of multivessel disease: a single-center matched-propensity controlled cohort study. Ann Thorac Surg. 2005; 79:1563-9. [PubMed: 15854934]

32. McNulty EJ, Ng W, Spertus JA, et al. Surgical candidacy and selection biases in nonemergent left main stenting implications for observational studies. JACC Cardiovasc Interv. 2011; 4:1020-7. [PubMed: 21939943]

33. Singh M, Rihal CS, Lennon RJ, Spertus JA, Nair KS, Roger VL. Influence of frailty and health status on outcomes in patients with coronary disease undergoing percutaneous revascularization. Circ Cardiovasc Qual Outcomes. 2011; 4:496-502. [PubMed: 21878670] 


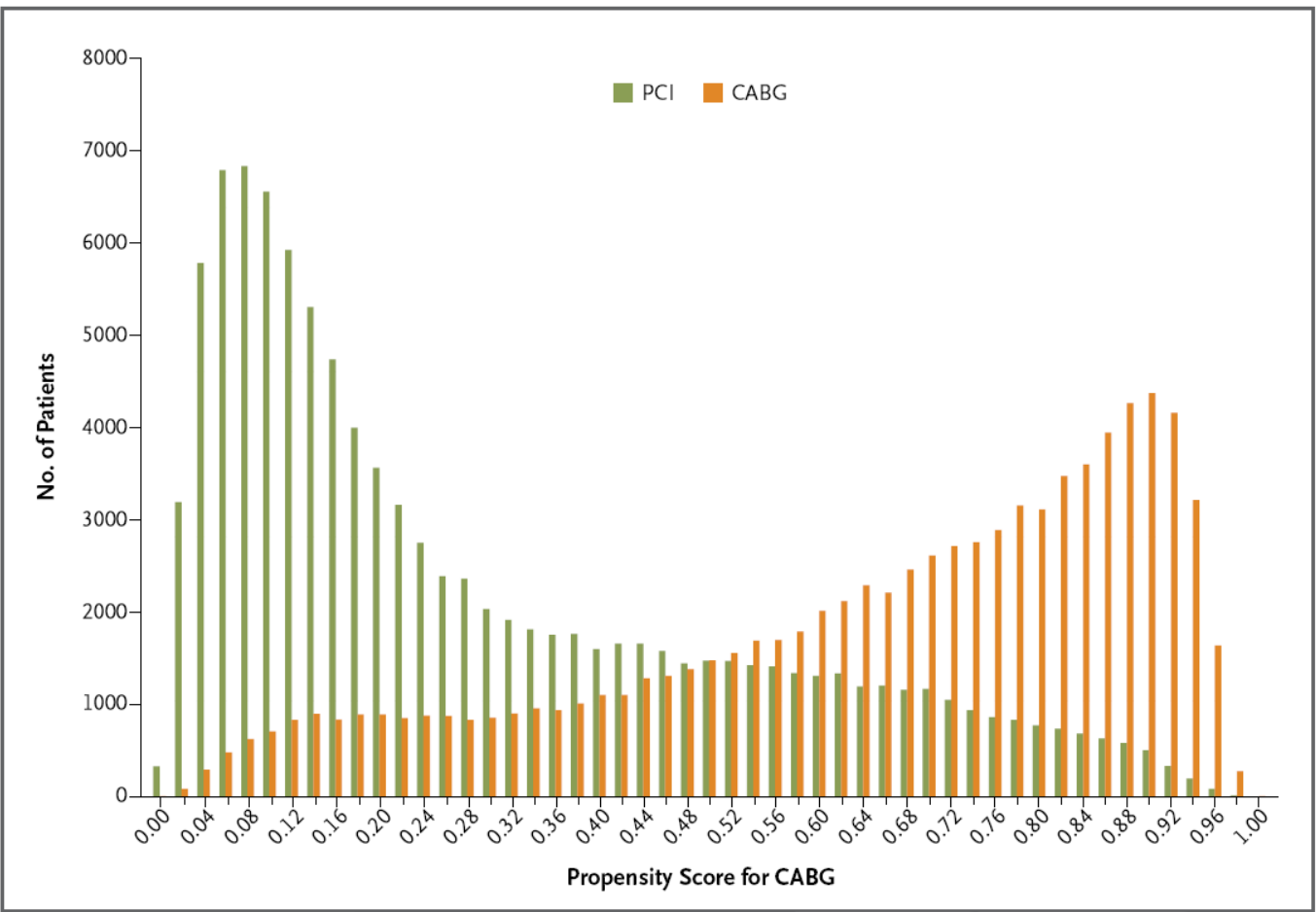

Figure 1. Propensity Scores for Coronary Artery Bypass Grafting (CABG) in the Percutaneous Coronary Intervention (PCI) and CABG Populations

The propensity score for $\mathrm{CABG}$ is the probability given baseline variables that any patient in either group would be selected for CABG. 


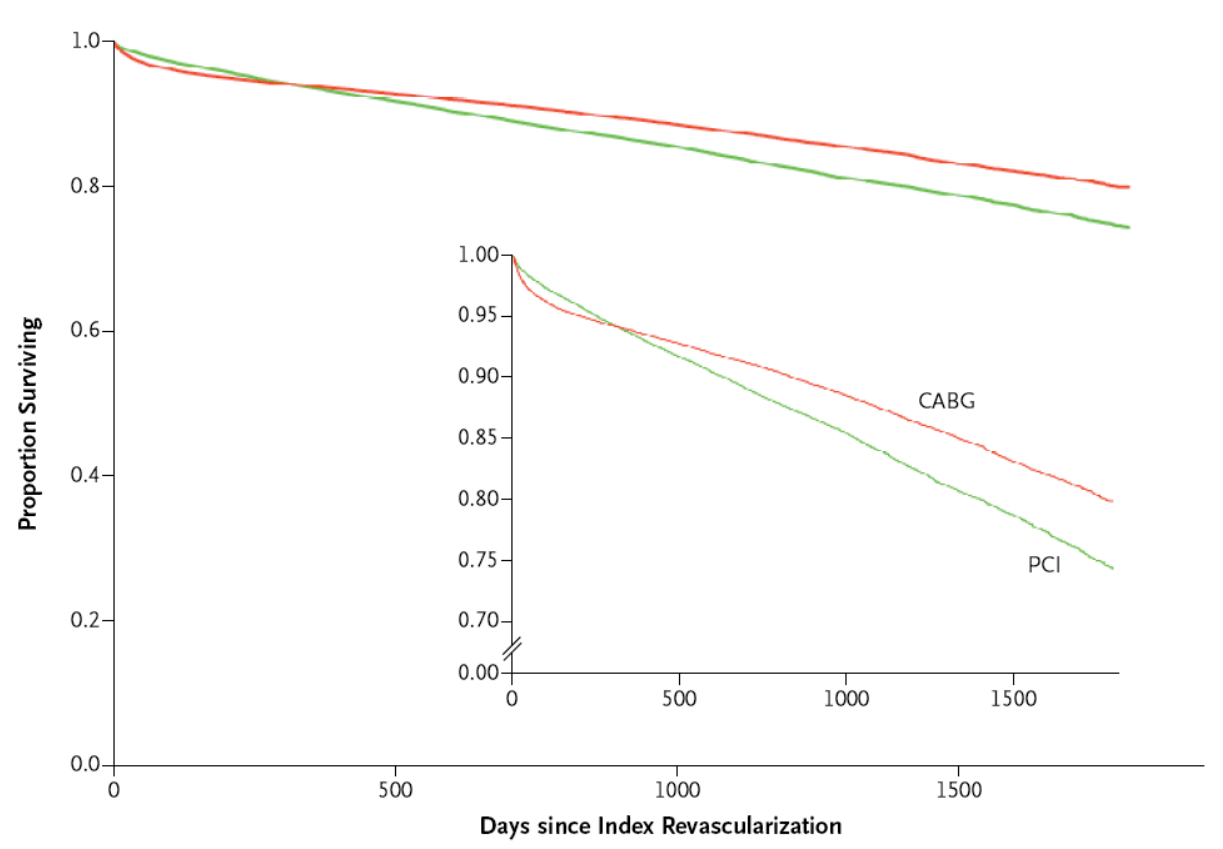

Mortality after CABG, \% (95\% CI) Mortality after $\mathrm{PCI}, \%(95 \% \mathrm{Cl})$ Relative risk with CABG $(95 \% \mathrm{Cl})$
30-Day

$2.07(1.98-2.17)$ $1.21(1.14-1.27)$ $1.72(1.58-1.84)$
$1-\mathrm{Yr}$

$6.00(5.58-6.17)$ $6.36(6.22-6.51)$ $0.94(0.91-0.97)$

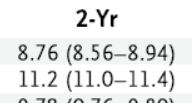

$0.78(0.76-0.80)$

$3-Y r$
$12.1(11.9-12.4)$
$16.0(15.7-16.2)$
$0.76(0.74-0.78)$

$4-\mathrm{Yr}$

$16.0(15.7-16.3)$

$20.9(20.6-21.3)$ $0.76(0.75-0.78)$

Figure 2. Rates of Survival in the CABG and PCI Populations, from an Unadjusted Analysis Cumulative mortality with CABG and with PCI and the relative risk of CABG as compared with PCI are shown. 


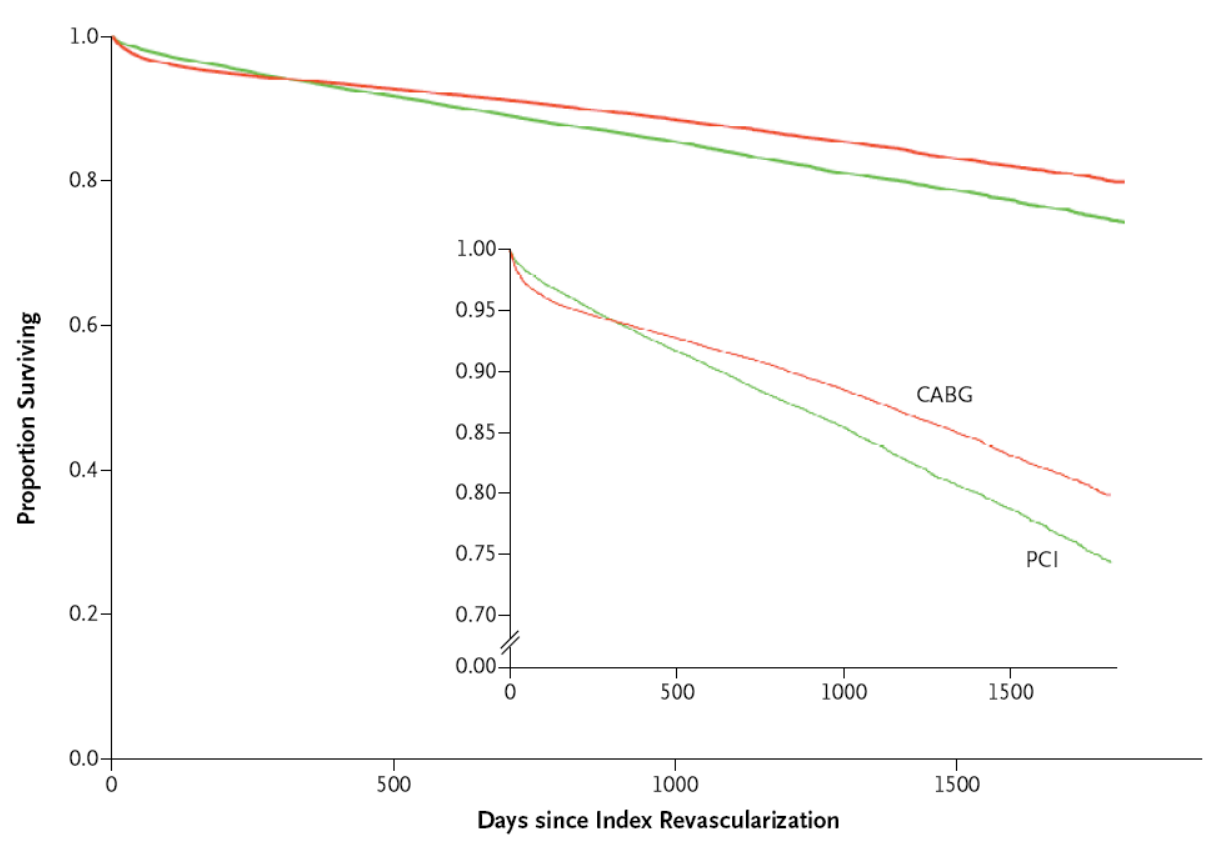

Figure 3. Rates of Survival in the CABG and PCI Populations, from an Analysis Adjusted with the Use of Inverse Probability Weighting

Cumulative mortality with $\mathrm{CABG}$ and with PCI and the relative risk of CABG as compared with PCI are shown. The inset shows the same data on an enlarged y axis. 


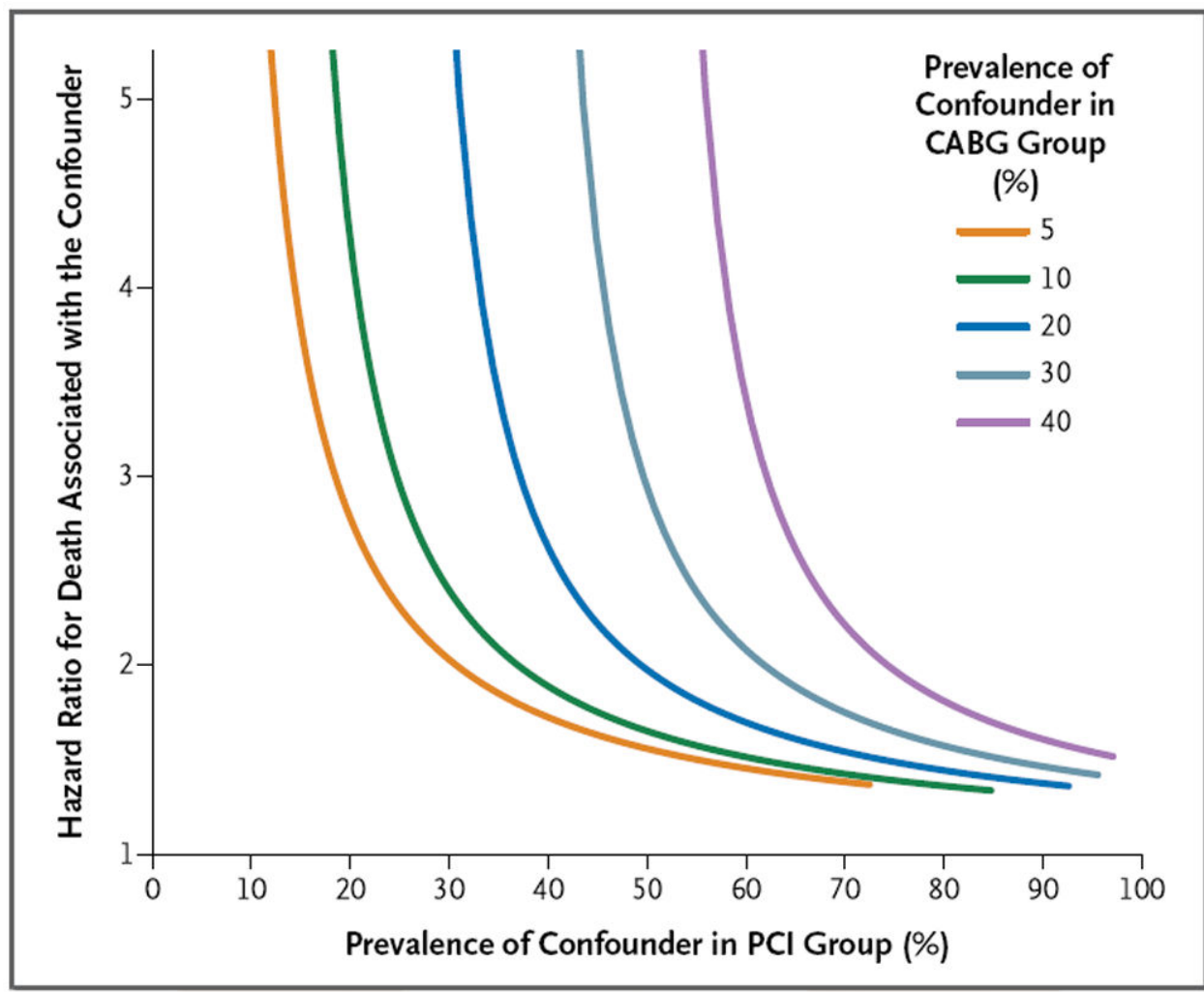

Figure 4. Effect of Unmeasured Confounding Factors

Shown is a sensitivity analysis that illustrates how powerful a single confounder would have to be to account for the advantage of CABG over PCI that was detected in the adjusted analysis. A single unmeasured confounder could produce the observed survival differences only if it increased the long-term risk of death by a factor of approximately two or if the long-term risk of death was three to five times as high in the PCI group as in the CABG group. For example, if a confounder was present in $10 \%$ of the patients in the CABG group (green curved line) but in $35 \%$ of patients in the PCI group (x axis), and if it increased the risk of death by a factor of slightly more than two (hazard ratio, 2.09), then that confounder alone could itself account for the observed difference in mortality between the study groups. 

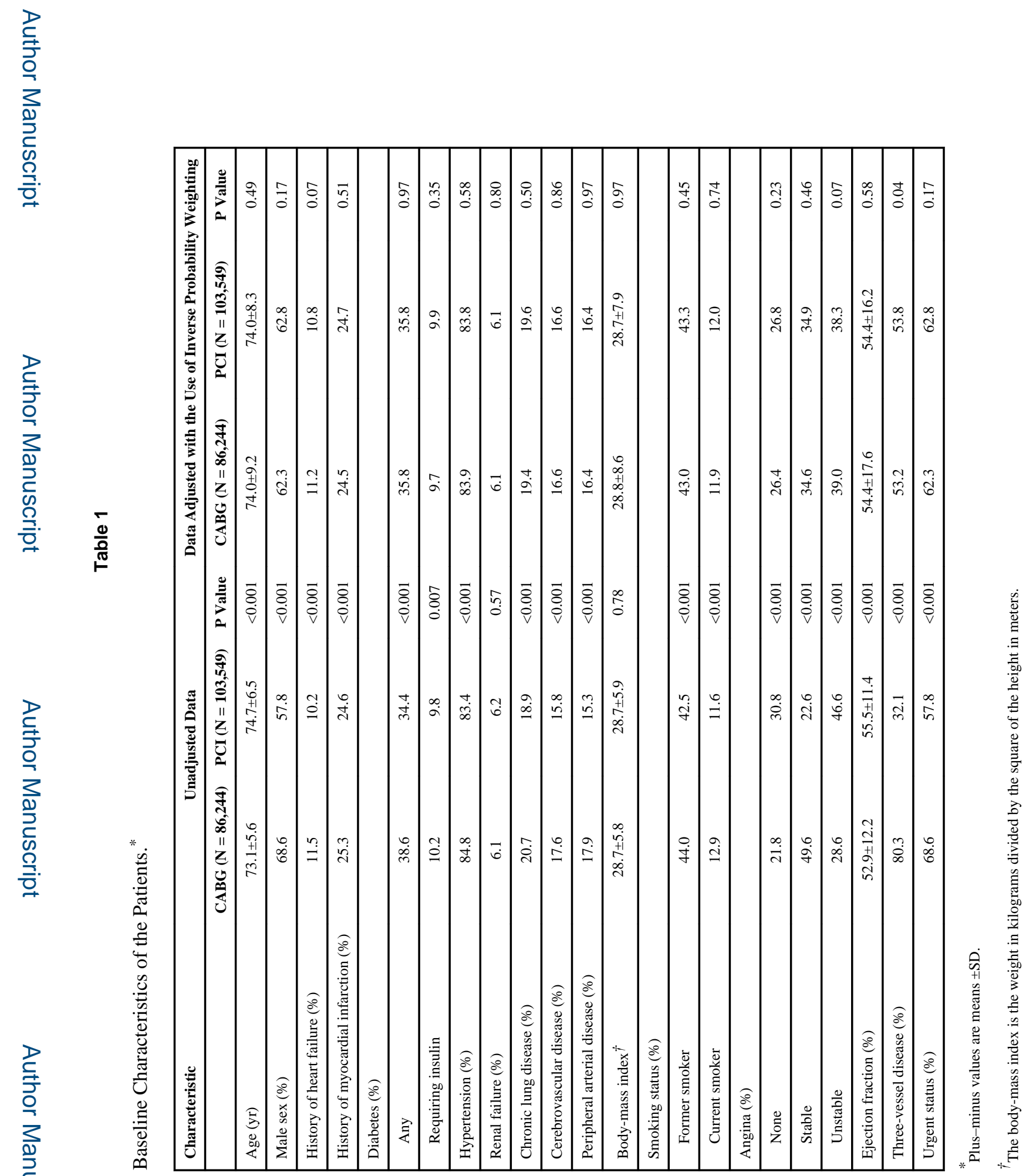



롬

N Engl J Med. Author manuscript; available in PMC 2015 December 07. 